\title{
$A b$ initio no core calculations of light nuclei and preludes to Hamiltonian quantum field theory
}

\author{
J.P. Vary*, P. Maris*, A.M. Shirokov*, , H. Honkanen*, J. Li*, S.J. \\ Brodsky $^{* *}$, A. Harindranath ${ }^{\ddagger}$ and G.F. de Teramond ${ }^{\S}$ \\ ${ }^{*}$ Department of Physics and Astronomy, Iowa State University, Ames, Iowa, 50011 USA \\ ${ }^{\dagger}$ Skobeltsyn Institute of Nuclear Physics, Moscow State University, Moscow, 119991, Russia \\ ** SLAC National Accelerator Laboratory, Stanford University, Menlo Park, California, USA \\ ${ }^{\ddagger}$ Theory Group, Saha Institute of Nuclear Physics, 1/AF, Bidhannagar, Kolkata, 700064, India \\ ${ }^{\S}$ Universidad de Costa Rica, San José, Costa Rica
}

\begin{abstract}
Recent advances in ab initio quantum many-body methods and growth in computer power now enable highly precise calculations of nuclear structure. The precision has attained a level sufficient to make clear statements on the nature of 3-body forces in nuclear physics. Total binding energies, spin-dependent structure effects, and electroweak properties of light nuclei play major roles in pinpointing properties of the underlying strong interaction. Eventually, we anticipate a theory bridge with immense predictive power from QCD through nuclear forces to nuclear structure and nuclear reactions. Light front Hamiltonian quantum field theory offers an attractive pathway and we outline key elements.
\end{abstract}

Keywords: nuclear structure, nuclear reactions, realistic Hamiltonians, three-nucleon potentials, light front Hamiltonian field theory

PACS: $21.60 . \mathrm{De}, 21.10 .-\mathrm{k}, 21.10 . \mathrm{Dr}, 21.30 . \mathrm{Fe}, 21.45 . \mathrm{Ff}, 11.15 .-\mathrm{q}$

\section{INTRODUCTION}

Non relativistic quantum many-body theory plays a leading role in several areas of physics. Recent advances in the microscopic ab initio theory of light nuclei provide a computational foundation for evaluating properties of light nuclei as well as properties of the hadrons and their interactions. The former employs realistic interactions that fit the nucleon-nucleon $(\mathrm{NN})$ phase shifts and properties of three-nucleon (NNN) systems. The latter employs a Hamiltonian from the underlying quantum field theory QCD that is quantized on the light front. The light front quantized Hamiltonian field theory appears similar to a non relativistic framework though it retains a covariant structure and features particle creation and annihilation.

\section{$A B$ INITIO NO CORE METHODS APPLIED TO LIGHT NUCLEI}

The "ab initio" problem in nuclear structure physics is to solve for nuclear properties with the best available NN potentials, supplemented by 3-body NNN potentials as needed using a quantum many-particle framework that respects all the known symmetries of the potentials. Among the few ab initio methods available for light nuclei beyond atomic number $A=10$, the no core shell model (NCSM) [1] and the no core full con- 
figuration (NCFC) [2] methods frame the problem as a large sparse Hamiltonian matrix eigenvalue problem.

Since NNN potentials enlarge the computational requirements by one to two orders of magnitude, we have demonstrated that similar results may sometimes be achieved by adjustments the off-shell properties of the NN potential. These adjustments preserve the original high precision description of the NN scattering data. A partial list of the NCSM and NCFC achievements includes:

- Described the anomaly of the nearly vanishing quadrupole moment of ${ }^{6} \mathrm{Li} \mathrm{[3]}$;

- Established need for 3-body forces to explain, among other properties, neutrino- ${ }^{12} C$ cross sections [4];

- Found quenching of Gamow-Teller (GT) transitions in light nuclei transitions due to combined effects of 3-body potential [5] (or off-shell modifications of NN potential) plus configuration mixing [6];

- Obtained successful description of $A=10$ to 13 low-lying spectra with chiral NN + NNN interactions [7];

- Explained level ordering of ${ }^{10} \mathrm{~B}$ by including chiral NNN interaction [7].

Here we focus on the NCFC method where we adopt the harmonic oscillator (HO) single particle basis which involves two parameters and we seek results independent of those parameters either directly in a sufficiently large basis or via extrapolation to the infinite basis limit. The first parameter $\hbar \Omega$ specifies the $\mathrm{HO}$ energy, the spacing between major shells. Each shell is labeled uniquely by the quanta of its orbits $N=$ $2 n+l$ (orbits are specified by quantum numbers $n, l, j, m_{j}$ ) which begins with 0 for the lowest shell and increments in steps of unity. Each unique arrangement of fermions (neutrons and protons) within the available HO orbits that is consistent with the Pauli principle, constitutes a many-body basis state. Many-body basis states satisfying chosen symmetries are employed in evaluating the Hamiltonian $H$ in that basis. The second parameter is $N_{\max }$ which limits the total number of oscillator quanta allowed in the many body basis states and thus limits the dimension $D$ of the Hamiltonian matrix. $N_{\max }$ is defined as the maximum of the total quanta above the minimum for the specific nucleus needed to satisfy the Pauli principle.

To illustrate the NCFC results, we adopt the realistic JISP16 NN potential from inverse scattering that provides an excellent description of the NN data [8]. This NN potential is sufficiently well-behaved that we may obtain NCFC results for light nuclei [2]. The left panel of Fig. 1 shows smooth uniform convergence of the ground state energy of ${ }^{6} \mathrm{He}$ in a sequence of $N_{\max }$ basis spaces. The space with $N_{\max }=16$ represents a Hamiltonian matrix with $D=595,922,646$ requiring about 20,000 processors for its solution.

The right panel of Fig. 1 presents the calculated upper limit to the ground state energy in each basis space as well as the extrapolations based on the preceding 4 ("extrapolation A") or 3 ("extrapolation B") basis spaces. Ref. [2] details the extrapolation methods. The present work extends Ref. [2] by including the $N_{\max }=16$ results and the comparison with a recent independent calculation using the hyperspherical harmonics method [6] with the same Hamiltonian. Note that the extrapolations are all consistent with each other since they lie within the assessed uncertainties. The overall conclusion is that JISP16, unlike other realistic NN interactions, produces a bound state of ${ }^{4} \mathrm{He}$ plus two neutrons. 

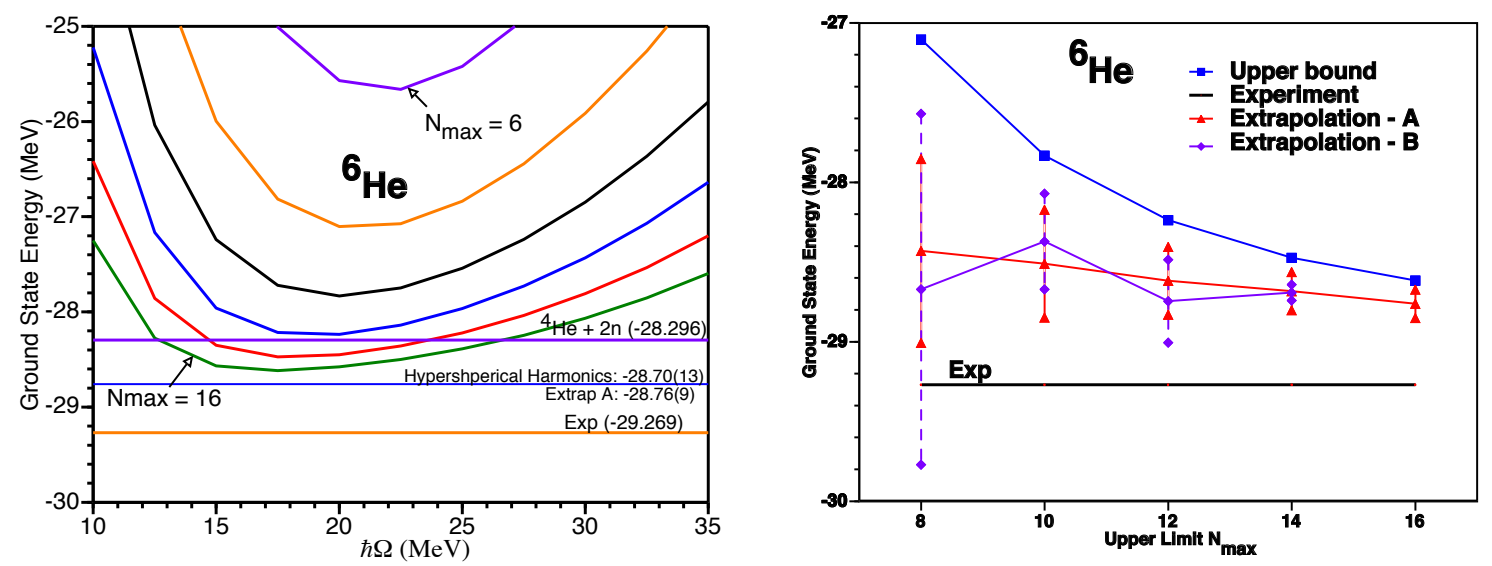

FIGURE 1. Left panel: ${ }^{6} \mathrm{He}$ evaluated in the NCFC approach that produces the extrapolated result indicated as "Extrap. A". The curves present the ground state eigenvalue as a function of $\hbar \Omega$ at a sequence of $N_{\max }$ values. The independent extrapolation of Ref. [6] is presented for comparison. Right panel: Upper bounds obtained at each $N_{\max }$ value and results of two extrapolation methods. The assessed uncertainties are indicated by the error bands on each extrapolated result.

However, it underbinds ${ }^{6} \mathrm{He}$ by about $500 \mathrm{keV}$.

\section{LIGHT FRONT HAMILTONIAN FIELD THEORY}

Recently, we have initiated investigations $[9,10]$ as preludes for solving Hamiltonian light front field theory nonperturbatively for QED and QCD. These are major efforts and we present here representative initial results. We introduced the Basis Light Front Quantized (BLFQ) field theory method, where we utilize basis space methods of the non-relativistic quantum many-body theory referenced above. For BLFQ, we adopt a 2-dimensional harmonic oscillator $(2 \mathrm{~d} \mathrm{HO})$ basis for the transverse modes and a discretized momentum basis for the longitudinal modes. The $2 \mathrm{~d} \mathrm{HO}$ basis is natural in light of the successful phenomenology of AdS/QCD [11]. Initially, we have introduced a cavity (harmonic oscillator confinement) in the transverse direction and enumerated the basis space states to gain an impression of the exponential buildup of dimensionality with increasing cutoffs. There are two cutoffs: the maximum $2 \mathrm{~d}$ HO quanta $N_{\max }$ (analogue of the $3 \mathrm{~d}$ quantity defined above) and $K$ the total light front momentum in units of $\frac{2 \pi}{L}$ where each parton takes one or more momenta and $L$ is the box length.

We present in Fig. 2 results from a model for a strongly interacting system in a cavity. We take the modes of QED for a system consisting of equal numbers of spin $\frac{1}{2}$ fermions and antifermions plus photons in a cavity. For specified cutoffs, we form the state that is an equally-weighted distribution of all allowed basis states (our strong coupling paradigm) and evaluate the light front momentum distribution fractions. The resulting fermion and boson distributions for cutoffs $N_{\max }=K=8$ are depicted in the left panel of Fig. 2 and illustrate the potential utility of the BLFQ approach. The right panel of Fig. 2 indicates the trends with increasing cutoffs, reminiscent of scale evolution. 

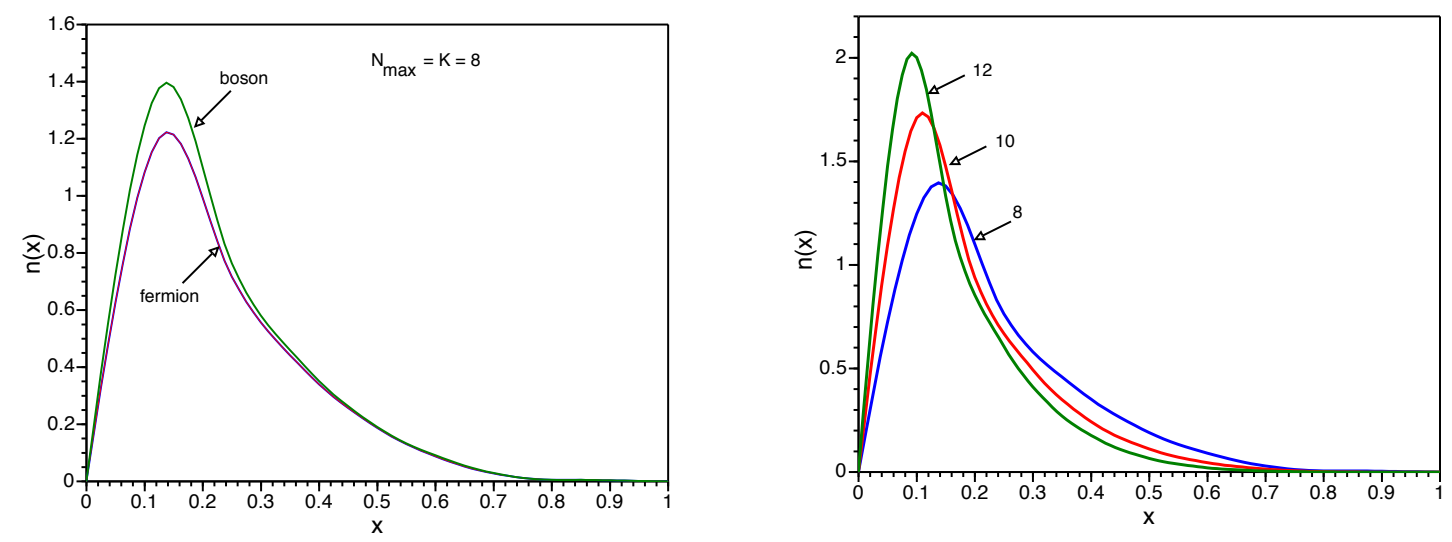

FIGURE 2. Light front momentum distribution functions for states representing a strong coupling paradigm. The antifermion distribution is the same as the fermion distribution. The total momentum fraction carried by the fermion plus antifermion distribution is 0.65 while the boson distribution carries the remaining fraction 0.35 . The right panel displays the boson distributions at three different values of $N_{\max }=K$ that are labeled.

\section{ACKNOWLEDGMENTS}

This work was supported by the US DOE Grants DE-FC02-09ER41582 and DE-FG0287ER40371 and by DOE Contract DE-AC02-76SF00515. This work was also supported in part by the Russian Foundation of Basic Research. Computational resources are provided by DOE through the National Energy Research Supercomputer Center (NERSC) and through an INCITE award (David Dean, PI).

\section{REFERENCES}

1. P. Navrátil, J. P. Vary and B. R. Barrett, Phys. Rev. Lett. 84, 5728 (2000); Phys. Rev. C 62, 054311 (2000).

2. P. Maris, J. P. Vary and A. M. Shirokov, Phys. Rev. C. 79, 014308(2009), arXiv 0808.3420.

3. P. Navrátil, J.P. Vary, W.E. Ormand, B.R. Barrett, Phys. Rev. Lett. 87, 172502(2001)

4. E. Caurier, P. Navrátil, W.E. Ormand and J.P. Vary, Phys. Rev. C 66, 024314(2002); A. C. Hayes, P. Navrátil and J. P. Vary, Phys. Rev. Lett. 91, 012502 (2003).

5. A. Negret, et al, Phys. Rev. Lett. 97, 062502 (2006).

6. S. Vaintraub, N. Barnea and D. Gazit, nucl-th 0903.1048

7. P. Navrátil, V. G. Gueorguiev, J. P. Vary, W. E. Ormand and A. Nogga, Phys. Rev. Lett. 99, 042501(2007); nucl-th 0701038.

8. A. M. Shirokov, J. P. Vary, A. I. Mazur and T. A. Weber, Phys. Letts. B 644, 33(2007); nucl-th/0512105.

9. J. P. Vary, H. Honkanen, J. Li, P. Maris, S. J. Brodsky, P. Sternberg, E. G. Ng and C. Yang, in Light Cone 2008 Relativistic Nuclear and Particle Physics, Proceedings of Science LC2008, 040 (2008); SLAC-PUB-13460; arXiv: 0812.1819.

10. J. P. Vary, H. Honkanen, J. Li, P. Maris, S. J. Brodsky, P. Sternberg, E. G. Ng and C. Yang, SLACPUB-13582; arXiv: 0905.1411; to be published.

11. G. F. de Teramond and S. J. Brodsky, Phys. Rev. Lett. 102, 081601 (2009) [arXiv:0809.4899 [hep$\mathrm{ph}]]$. 